\title{
NUEVAS FORMAS DE RACISMO: ESTADO DE LA CUESTIÓN EN LA PSICOLOGÍA SOCIAL DEL PREJUICIO
}

\author{
NEW RACISMS: \\ STATE OF THE ART IN THE SOCIAL PSYCHOLOGY OF PREJUDICE
}

\author{
Pablo Pascale \\ Universidad de Salamanca, España
}

\begin{abstract}
Resumen: ¿Por qué somos racistas? En la actualidad, la amplia mayoría de la gente responderá que no es racista. Y es posible que no lo sea, al menos, conciente o voluntariamente. Durante los últimos años, la Psicología Social ha estado investigando que el racismo ya no se expresa de manera frontal, explícita. El racismo de hoy es encubierto, latente, ha cambiado y se ha adaptado al control social, se oculta bajo nuevas formas de expresión lingüística, creencias y actitudes. En la era de lo políticamente correcto, el racismo se sanciona, por ello se amolda y continúa presente sin ser confrontativo. Esto es lo que los psicólogos sociales han dado en llamar el nuevo racismo.
\end{abstract}

Palabras clave: Prejuicio, discriminación racial y étnica, nuevo racismo, Psicología Social.

\begin{abstract}
Why are we racists? Nowadays, most people will answer they are not racists; and that is possible, at least consciously or intentionally. During the past few years research in social psychology has shown that racism is no longer expressed in a frontal or explicit manner. Today racism is latent, more subtle, has changed and adapted to social control, hidden under new forms of linguistic expressions, beliefs and attitudes. In the era of the "politically correct", racism is sanctioned, and for that reason it moulds without confrontations. This is what social psychologists call the new racism.
\end{abstract}

Keywords: Prejudice, racial and ethnic discrimination, new racism, social psychology.

\section{INTRODUCCIÓN}

Si bien se han realizado avances políticos y sociales significativos, tales como la aplicación de políticas de acción afirmativa o el cambio de denominación en Estados Unidos de black a african american, o en América Latina de negro a afro-descendiente, el racismo aún continúa existiendo. Prueba de ello es que las diferencias sociales entre etnias, o entre autóctonos e inmigrantes, continúan siendo evidentes. A modo de ejemplo, salvo escasas excepciones, la población afro-descendiente en América Latina constituye la parte de la población con peores indicadores de ingreso, empleo y educación; está pobremente representada en los gobiernos, los parlamentos y en los directorios de empresas; además, es invisible para censos y encuestas de hogares de varios países de la región (Antón \& Del Popolo, 2008; Banco Mundial, 2004, 2008; Bello \& Paixao, 2008).
O asimismo, un estudio realizado en las 100 compañías mas grandes de Europa ha revelado que muy pocas tienen empleados en posiciones intermedias, y ninguna contaba con un CEO miembro de minorías étnicas (Foroohar, 2002).

Se observa que aún hoy la discriminación persiste, que se ha transformado, ha desarrollado mecanismos más sutiles y difíciles de identificar. Esto ha impulsado a los psicólogos sociales a investigar estas nuevas modalidades de ser racista.

En la presente comunicaciòn se explora el estado de la cuestión de las nuevas formas de racismo, desde la perspectiva de la Psicología Social. Pero es necesario, para referir a las nuevas formas de racismo aludir al racismo tradicional, principalmente porque muchas personas no se consideran racistas, por el solo hecho de no identificarse con postulados del racismo tradicional, lo cual no significa que no lo sean. 
En los estudios de la Psicología Social del Prejuicio y racismo, se pueden identificar dos grandes momentos. El primero comienza con la publicación de The nature of prejudice de Allport en 1954. Aquí se concentran los estudios que construyen modelos y Teorías del Prejuicio, que procurarán explicar el racismo clásico.

Mientras que, el segundo momento, comienza en la década de los 80 con la emergente idea de nuevo racismo, que ocupa la atención de los psicólogos sociales actualmente. Principalmente, son Kinder y Sears (1981) y McConahay (1986) quienes observan que en la sociedad norteamericana surge un manifiesto rechazo por las creencias racistas, lo que se traduce en menores índices de expresiones Prejuiciosas y racistas. Sin embargo, el racismo no desaparece sino que cambia, se transforma en más sutil y subyacente, más difícil de identificar y medir (Dovidio \& Fazio, 1992; Pettigrew, 1998).

En este trabajo exploraremos brevemente la primera etapa en la que se construyen los cimientos conceptuales del Prejuicio y racismo tradicional, que hoy nos sirven para comprender las nuevas formas de racismo, que exploraremos con más detenimiento en su estado actual.

\section{LA PSICOLOGÍA SOCIAL Y EL PREJUICIO}

Previo a ingresar en la primera etapa de estudios sobre el Prejuicio y racismo, debemos preguntarnos qué aporta la Psicología Social como disciplina científica al avance de esta temática.

El primer avance en los estudios psicológicos del tema fue realizado por Allport (1954), quien pasó de concebir al Prejuicio como una expresión individual atribuida a determinados rasgos de personalidad, a comprender el Prejuicio como un fenómeno intergrupal. Un fenómeno que puede ser analizado en individuos actuando como miembros de un grupo es, en última instancia, el objeto de la Psicología Social.

En forma muy sintética, podemos decir que la Psicología Social es una disciplina que estudia la interacción entre el individuo y los grupos sociales (Tajfel \& Fraser, 1978). Si se quiere, tal como especificara Munné (1986) el comportamiento interpersonal implica formalmente una relación, que conductualmente se da como una interacción, productora de unos efectos consistentes en la influencia social. O lo que es lo mismo, el objeto de la Psicología Social consiste en el estudio, a nivel de las relaciones interpersonales, de la interacción a través de sus efectos.

Por lo tanto, el Prejuicio y racismo se dan en la interacción entre individuos como pertenecientes a grupos, y en el campo de esta interacción la Psicología Social realiza sus aportes.

De allí que, establecer que el Prejuicio es un fenómeno intergrupal es el primer gran aporte de la Psicología Social a este campo, pasando a considerar que el Prejuicio se genera en procesos de grupo, que tienen sus factores causales en las percepciones de los individuos y su comportamiento hacia miembros de otros grupos (Brown, 1995). Igualmente, se reconoce que los principios psicológicos por sí solos no son suficientes para explicar el surgimiento de actitudes racistas, puesto que las mismas deben ser comprendidas en términos de su contexto histórico, económico y político. Estos procesos sociales son los que influencian a los procesos psicológicos, y sobre los que la Psicología Social hace sus estudios.

\section{APROXIMACIÓN BIBLIOMÉTRICA SOBRE PREJUICIO Y DISCRIMINACIÓN}

En todo estudio sobre el estado actual de una temática, se hace preciso visibilizar el panorama de producción científica del ámbito. Aquí hemos realizado una aproximación bibliométrica al volumen de publicaciones que se realizan en psicología sobre el Prejuicio y la discriminación.

Basado en la evidencia de que no existen estudios que exploren la producción científica en psicología, sobre este ámbito para el período 1980-2007, a continuación se explora brevemente el volumen actual de esta producción y su tasa de crecimiento respecto a los últimos años. A su vez, se observa qué tipo de discriminación es la que concentra el mayor número de publicaciones. De esta forma, se busca responder a las 
siguientes preguntas: ¿Cuanto se ha escrito sobre Prejuicio y discriminación en los últimos 28 años? ¿Están aumentando significativamente las publicaciones sobre este campo?

El método utilizado para analizar y cuantificar el volumen de publicaciones de Prejuicio y discriminación ha sido mediante la base informatizada del Psychological Abstracts (PsycINFO), ingresando los descriptores prejudice y social discrimination para el período comprendido entre 1980 y 2007 . Los descriptores son listados por la base de datos para indicar los contenidos de cada publicación y, en este caso, son asignados según el Tesauro de PsycINFO para aquellas publicaciones cuyo contenido concierne a Prejuicio o discriminación social, esta última entendida como trato Prejuicioso o diferenciado basado en la religión, raza, minusvalía u otras características no fundadas en el mérito.

Dado que el propósito del artículo es presentar la actualidad de la temática del Prejuicio y la discriminación, se ha acotado el período a los últimos años. El año 2008 no ha sido incluido en el período, porque la base de datos continúa registrando publicaciones para el último año durante los primeros meses del siguiente (2009).

La búsqueda en PsycINFO, de los descriptores Prejuicio o discriminación social informa de 3999 publicaciones en el periodo 1980-2007.
En el grafico 1 se aprecia un sustantivo incremento de publicaciones, fundamentalmente, a partir del año 2001. Se ha ajustado un modelo de crecimiento exponencial de publicaciones que es capaz de explicar un $87.29 \%$ de la variabilidad temporal de la serie (ver apéndice 1).

A este respecto, existen diversas formas de estimar si el crecimiento de publicaciones en un período es significativo, entre ellas su tasa de crecimiento interanual comparado a otro ámbito de conocimiento (Pascale, 2008). Dados los objetivos del presente artículo hemos optado por establecer una comparación entre las tasas de crecimiento interanual de Prejuicio y discriminación en relación al volumen total de publicaciones en psicología para la base de datos PsyclNFO, para el período 1980-2007.

En el caso del Prejuicio y la discriminación, los datos arrojan un $10,04 \%$ de tasa de crecimiento interanual, mientras que la Psicología en general experimenta un $5,3 \%$. Esto muestra que la temática del Prejuicio es de interés creciente dentro de los estudios en psicología.

En suma, el campo de estudios del Prejuicio y la discriminación experimenta un crecimiento exponencial de publicaciones para los últimos 28 años, y dicho crecimiento es significativo en comparación al volumen total de publicaciones en psicología.

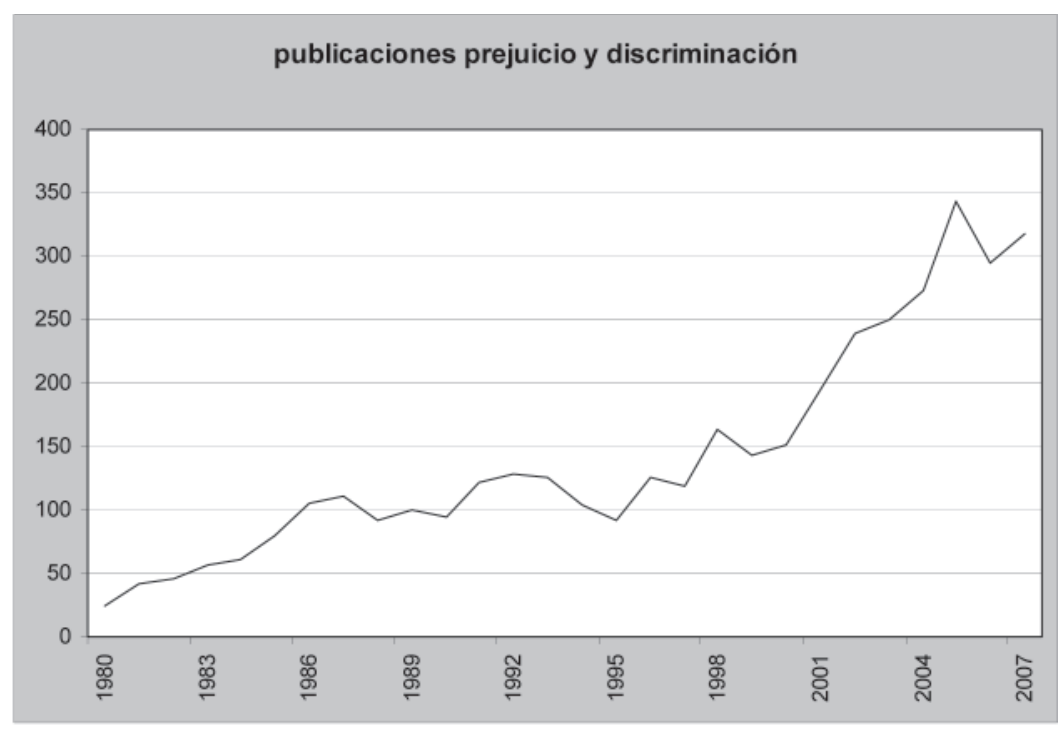

Grafico 1. Publicaciones de Prejuicio y discriminación para período 1980-2007 


\section{MOMENTO 1. LAS BASES PARA COMPRENDER EL PREJUICIO}

A nivel psicológico y psicosocial, el racismo funciona sobre la base del proceso de categorización de las personas, atribuyéndoles características propias de determinado grupo. Esto se complementa con la identificación del individuo con un grupo, que considerará superior que otros a los que se compara.

De esta forma, los tres conceptos principales sobre los cuales la Psicología Social construye su análisis del Prejuicio y la discriminación racial son: categorización social, estereotipo e identificación social. Si bien, estos tres conceptos influenciaron los estudios de la primera etapa de producción científica, continúan vigentes en los estudios actuales de las nuevas formas de racismo.

\section{Categorización social}

Tal como lo propuso Allport (1954), la categorización social es un mecanismo cognitivo normal, y es el precursor de toda forma de Prejuicio. Por lo que, la categorización por sí misma no es suficiente para explicar el Prejuicio. Tajfel y Turner (1986) delimitaron su alcance al indicar que la categorización es una herramienta cognitiva que ayuda al ser humano a segmentar, clasificar y ordenar la realidad, pues en caso contrario sería incapaz de reaccionar de una forma específica a cada estímulo que se le presenta.

Los procesos cognitivos que guían a este mecanismo son la "asimilación" y la "diferenciación". Mientras que la asimilación refiere al efecto de minimizar las diferencias dentro de una categoría, la diferenciación es el efecto de exagerar las diferencias preexistentes entre categorías. (Tajfel, 1959). En términos de la Psicología Social, esto significa que los miembros de diferentes grupos tienden a verse más diferentes de lo que realmente son, y los miembros de un mismo grupo más similares entre ellos (Eiser \& Stroebe, 1972; Krueger \& Clement, 1994; Tajfel \& Wilkes, 1963).

Un tercer proceso cognitivo de la categorización, muy cercano a la asimilación, es el efecto de "homogeneidad del grupo externo", en el que la gente tiende a ver a los miembros del grupo externo (exogrupo) más similares entre ellos que, a miembros del grupo interno (endogrupo).

Este efecto de percepción de igualdad se mantiene, sin importar si el exogrupo es de raza, religión, nacionalidad, u otros (Linville, 1998).

Se ha hallado que la categorización es un proceso cognitivo del que los niños a partir de los 3 años ya hacen uso, y son conscientes de las dos principales categorías sociales: etnia y género (Duveen \& Lloyd, 1986; Thompson, 1975; Williams \& Morland, 1976; Yee \& Brown, 1994). Inclusive, se ha estudiado que desde esa edad, los niños comienzan a desarrollar preferencias actitudinales entre estas categorías, identificándose con miembros de su propio grupo étnico (Aboud, 1980) o de género (Thompson, 1975; Yee \& Brown, 1994). Ésta discriminación se expresa con un favoritismo endogrupal (Yee \& Brown, 1994).

En tal sentido, los efectos directos de la categorización social, de interés para la explicación del Prejuicio, son la exageración de las diferencias entre categorías y la disminución de diferencias, al interno de las mismas. Una vez que se exageran las diferencias entre el "nosotros" y el "ellos", el efecto complementario es "ellos son todos iguales, en cambio nosotros tenemos nuestras características que nos diferencian".

\section{Estereotipo}

De esta forma, surge el estereotipo, entendido como la atribución a una persona de características que se consideran como pertenecientes a todos, o casi todos, los miembros de un grupo.

La relación entre categorización social y estereotipo es que éste último es una inferencia inspirada en la asignación de una persona a una categoría determinada (Brown, 1995). Esto significa que el estereotipo es la percepción de que la mayor parte de los miembros de una categoría comparte los mimos atributos, por lo que el estereotipo deviene directamente del proceso de categorización.

Se ha hallado que hay, al menos, tres fuentes del estereotipo: a) la transmisión sociocultural 
mediante la familia, escuela y medios de comunicación (Allport, 1954); b) otra es la que se ha denominado como "aire de verosimilitud", entendiendo que el estereotipo deviene de algunos aspectos de la realidad social, como diferencias socioeconómicas o culturales (Brewer y Campbell, 1976) y; c) el sesgo cognitivo de "correlación ilusoria", mediante el cual dos variables son asociadas entre ellas, cuando en realidad hay muy poca o ninguna asociación.

Habitualmente, se entiende que los sucesos menos frecuentes atraen una mayor cantidad de nuestra atención y, por ello, son recordados con mayor facilidad (Hamilton y Gifford, 1976). A modo de ejemplo, en un país predominantemente blanco se recordarán más fácilmente las conductas antisociales cometidas por negros, que las cometidas por blancos. La correlación ilusoria está en establecer una correlación entre la delincuencia y el color de piel.

\section{Identificación social}

La identificación social es el tercer concepto sobre el que la psicología ha asentado sus estudios del Prejuicio y racismo. Partiendo del concepto de categorización social, Tajfel y Turner (1986) desarrollan la Teoría de la identidad social, que sirve a efectos de explicar dos procesos necesarios para comprender el Prejuicio, y por tanto, la discriminación: el favoritismo del grupo interno y, la necesidad de autoestima.

La identidad social es definida como los aspectos del autoconcepto de una persona que se derivan de su pertenencia a grupos (Tajel y Turner, 1986). Estos autores infieren que las personas tienden a verse a sí mismas en forma más positiva que negativa, por lo que si nuestra identidad está determinada por nuestra pertenencia a un grupo, entonces, tendremos una preferencia por ver a nuestro grupo en forma más positiva, que a otros grupos a los que no pertenecemos. Esto significa que las personas mantienen su autoestima, en buena parte, por su identificación con grupos. Para lograrlo, es preciso comparar el endogrupo y el exogrupo en aquellas dimensiones relevantes que resulten en una evaluación favorable al endogrupo.

Se ha hallado que las personas con baja autoestima, tienen más predisposición a expresar
Prejuicios (Fein \& Spencer, 1997), con lo cual, el Prejuicio cumpliría la función de mantener su autoestima.

Por lo tanto, para comprender el Prejuicio es preciso señalar que no solo los sentimientos y actitudes negativas hacia el exogrupo son los que intervienen en el Prejuicio y discriminación, sino también el favoritismo hacia el grupo propio, mecanismo que ha quedado demostrado para diferentes contextos culturales (Aberson, Healy \& Romero, 2000; Brewer, 1979, 1999).

\section{Desarrollo del Prejuicio}

Estos tres conceptos son los que permiten, en la primera etapa de estudios, describir las bases psicosociales sobre las que funciona el Prejuicio. A su vez, éstos aportan un marco para identificar las situaciones que potencian el desarrollo del Prejuicio, a saber: la ocupación cognitiva y emocional, la competencia por recursos, la amenaza a la identidad social y la deprivación relativa.

Gilbert y Hixon (1991) encontraron que los Prejuicios realmente funcionan como atajos mentales para ahorrarnos trabajo, cuanto más ocupados estamos por una tarea cognitiva, más recurriremos a los Prejuicios para relacionarnos o interactuar. Lo mismo sucede si estamos ocupados emocionalmente (Stephan \& Stephan, 1985), lo cual se ha demostrado mediante estudios experimentales que informan que, ante las emociones intensas aumentan las probabilidades de establecer juicios estereotipados (Mackie et al, 1996; Wilder \& Shapiro, 1989;).

Por su parte, cuando dos grupos compiten por el reparto de recursos, presentan una mayor probabilidad de desarrollar Prejuicios uno con respecto al otro. En cambio, disminuye la probabilidad, cuando ambos grupos cooperan por alcanzar conjuntamente un objetivo en común.

La relación entre la competencia por recursos y el desarrollo de Prejuicios ha sido elaborada por la "Teoría del grupo realista" (LeVine \& Campbell, 1972).

A su vez, los Prejuicios se ven potenciados cuando ante la amenaza a la identidad social de una persona, ésta responde intensificando su percepción positiva por el endogrupo y negativa 
por el exogrupo (Giles, 1977). La amplia variedad de amenazas, por ejemplo, a los valores y estatus económico del endogrupo, crean una ansiedad intergrupal que fomenta el Prejuicio (Duckitt, 2001).

Por último, el vínculo entre deprivación relativa y Prejuicio racial ha quedado demostrado (Pettigreww et al, 2008). Gurr (1970) propone que la deprivación relativa surge cuando las personas perciben una discrepancia entre el estándar de vida que tienen y el que creen que deberían tener. O sea, una brecha entre los logros actuales y las expectativas (Brown, 1995). En términos grupales, cuando percibimos que otro grupo lo está haciendo mejor o peor que el nuestro y, por ello, nos sentimos deprivados o gratificados.

Han sido, entonces, la categorización social, el estereotipo y la identificación social, las que han influenciado hasta nuestros días la perspectiva psicosocial del Prejuicio, y que han permitido definirlo como actitudes negativas dirigidas hacia personas por el hecho de pertenecer a un grupo determinado (Augoustinos \& Reynolds, 2001).

Si realizáramos una distinción entre Prejuicio y discriminación, se podría especificar que esta última se describe como una conducta injusta o un trato desigual a otras personas sobre la base de su pertenencia a un grupo, en tanto el primero se refiere habitualmente a las actitudes negativas hacia grupos desfavorecidos y sus miembros (Dion, 2002).

Por tanto, el Prejuicio es una actitud que tiene tres componentes principales: creencias o cogniciones del objeto (persona como perteneciente a un grupo), afectos o emociones que activan sentimientos positivos o negativos hacia el objeto, y la suma de ambos componentes genera un comportamiento hacia los grupos. En Psicología Social esta dimensión conductual del Prejuicio es la que se conoce como discriminación.

La discriminación así concebida, ha sido útil para la descripción del racismo clásico, entendido como sentimientos de odio injustificados por miembros de razas diferentes a las de uno mismo, teniendo como resultado el insulto, la exclusión, la discriminación, y en algunos casos, la violencia (Zack, 2002).
Como Vala et al, (2009) exponen, durante el siglo XX se ha asistido a una transformación gradual en la naturaleza del racismo, pasando de un racismo clásico a uno moderno o nuevo.

Como veremos más adelante, los mecanismos de activación del Prejuicio (categorización social, estereotipo e identificación social) continúan siendo los mismos, pero lo que cambia es su manifestación conductual expresada en nuevas formas de discriminación racial.

\section{MOMENTO 2. EL NUEVO RACISMO}

La mayoría de los psicólogos sociales concuerdan en que, los cambios de normativas sociales acaecidas en los últimos 50 años con las que se sancionan las expresiones racistas, han derivado en el desarrollo de formas más subyacentes, encubiertas, y simbólicas de expresión del Prejuicio hacia exogrupos.

Esto no significa que la forma tradicional de racismo haya desaparecido. De hecho, continúa siendo expresada en las sociedades occidentales democráticas (Walker, 2001). A modo de ejemplo de esto último, la Encuesta Social Europea-1 -llevada a cabo en 2002-, informa que el $52 \%$ de los europeos se oponen a la inmigración de personas de diferente raza o grupo étnico, o que el $27 \%$ opinan que no les gustaría que una persona de otra raza o grupo étnico contraiga matrimonio con un pariente cercano.

Si bien la forma clásica de racismo aún perdura, se ha evidenciado que desde hace unos 20 años ha disminuido considerablemente (Dovidio \& Fazio, 1992; Jackson, Brown \&Kirby, 1998; Pettigrew, 1998), y se ha tornado más difícil de detectar por la emergencia de las formas nuevas de racismo, como hemos visto, más encubiertas y simbólicas. A modo de ejemplo, en términos de racismo anti-negros, recientes investigaciones han hallado que el núcleo central del nuevo racismo se expresaría en que las personas blancas probablemente expresarán Prejuicios anti-negros siempre que les sea posible negarlos (Dovidio \& Gaertner, 2000; Hodson, Dovidio \& Garetner, 2002).

Previo a presentar estas nuevas formas, conviene realizar una precisión sobre el con- 
cepto de "raza", que se ha dado en naturalizar y aceptar como verdadero, inclusive por el discurso científico, al momento de clasificar a grupos sociales. Desde hace más de 50 años, el concepto de raza ha comenzado a ser desacreditado por los genetistas (Richards, 1997), y ya en 1951 la UNESCO lo estima un concepto inválido científicamente, por constituir un constructo social sin fundamento social. Finalizando este ciclo, en el año 2001 se completó el genoma humano, en el que a partir del análisis genético de 5 personas de diferente etnia, se concluyó que no era posible diferenciar una etnia de la otra, por lo que la "raza" no era un constructo biológico o científico válido (Gannett, 2001).

El motivo de la supervivencia del concepto de raza a pesar de la evidencia científica contraria es, justamente, su naturalización como categoría para clasificar a las personas (Augoustinos, 2009).

Inclusive, su persistencia se puede manifestar en la adopción de nuevas formas socialmente más aceptadas. A modo de ejemplo, en América Latina el cambio de denominación de negro a afrodescendiente, si bien ha sido un avance, ha desplazado el acento de raza a etnia, aunque posiblemente no haya desplazado aún las representaciones sociales existentes que se anclan en el racismo.

Una vez que el racismo clásico comienza a tornarse más difícil de detectar en nuestras sociedades, la Psicología Social identifica las emergentes formas de nuevo racismo que, más sofisticadamente, se presentan en las interacciones grupales.

Las nuevas formas de racismo han recibido una amplia variedad de denominaciones, entre ellas: racismo simbólico (Kinder \& Sears, 1981, Sears, 1988), racismo aversivo (Dovidio \& Gaertner, 2000), racismo moderno (McConahay, 1983), racismo ambivalente (Katz, 1981), ontologización (Roncarati et. al, 2009), Infrahumanización (Leyens et al, 2003), heteroetnización (Vala et al, 2009).

La mayoría de estas denominaciones comparten como elemento común que el nuevo racismo es tal cuando, en contexto sociales donde se promueve el anti-racismo, se practican formas indirectas de atribución de inferioridad a exogrupos (Vala, 2009). En este sentido, el nuevo racismo refiere a aquellas actitudes, comportamientos y creencias encubiertas que colocan al "otro" en la de una categoría humana inferior.

Asu vez, cada una de estas denominaciones refiere a formas específicas de expresión del racismo, que veremos aquí.

\section{Racismo simbólico y racismo moderno}

A principios de la década de los 80 Kinder y Sears (1981) y McConahay (1983) desarrollaron la idea de que en Estados Unidos estaba surgiendo una nueva forma de racismo. Este, era una "mezcla de afecto anti-negro y la defensa de los valores morales tradicionales americanos, que están personificados en la ética protestante" (Kinder \& Sears, 1981, p. 416). El núcleo de su Teoría estriba en que, las Teorías étnicas violan estos valores morales norteamericanos como el individualismo, la confianza en sí mismo, la ética del trabajo, la obediencia y la disciplina.

McConahay (1983) informa que el racismo simbólico o moderno tiene tres características: a) discrepancia contra los negros que "empujan muy fuerte" y ascienden rápido; b) discrepancia hacia las concesiones hechas a los negros, tales como la "discriminación positiva"; c) la negación de que exista una discriminación racial.

Sears (1988) explica que la gente sufre de respuestas emocionales condicionadas ante "símbolos" como los negros o los inmigrantes, asociándolos a hechos negativos. De esta forma, el racismo simbólico, se caracteriza por rechazar el racismo tradicional pero, simultáneamente, expresar un Prejuicio indirecto, como puede ser el caso de oponerse a la implementación de políticas de acción afirmativa para minorías raciales (Henry \& Sears, 2002; Kinder \& Sears, 1981; Sears, 1988).

\section{Racismo ambivalente}

El racismo ambivalente, se expresa en forma similar al simbólico y moderno, pero aquí se activa con la experimentación de un conflicto emocional entre sentimientos negativos y positivos hacia determinados grupos raciales (Katz, 1981). El conflicto emocional 
surge por la tensión entre dos valores morales: el reconocimiento de la igualdad democrática, y el individualismo. Las personas tienden a oscilar entre estos dos extremos y mostrar hacia otra etnia o bien una exagerada simpatía o bien una incomodidad.

Esto es lo que se conoce como amplificación de respuesta, y se expresa en extrema bondad hacia otra etnia, o lo contrario.

\section{Racismo aversivo}

Por su parte, el racismo aversivo ha recibido mucha atención por parte de las investigaciones en Psicología Social. El mismo se puede resumir como el respeto y creencia de los principios igualitarios para todas las razas, pero demostrando una aversión personal hacia las minorías (Gaertner \& Dovidio, 1986). Esta aversión personal se expresa en sentimientos de evitación (miedo, ansiedad, incomodidad) frente al otro.

Tal como se ha demostrado experimentalmente (Dovidio, 2001), una de las características principales de esta forma de racismo es que el racista aversivo discrimina cuando puede atribuir su conducta a cuestiones relacionadas con el grupo étnico de la persona que sufre el Prejuicio.

Esta forma de racismo es especialmente perjudicial dado lo complejo y encubierto de su lógica: por un lado el racista aversivo simpatiza con la víctima del Prejuicio por el trato injusto que ha recibido en el pasado, y apoya las políticas que vayan contra el racismo, y por otro lado, mantienen sentimientos y creencias negativas sobre los integrantes de otras etnias.

Los autores reflexionan que esta clase de racismo está presente en muchas personas blancas liberales que creen sinceramente no ser prejuiciosas. A este respecto, Dovidio et al (1997) hallaron que las formas de racismo simbólico y moderno, se correlaciona mayormente con una perspectiva política conservadora, mientras que el racismo aversivo estaría más presente entre liberales políticos.

Las actitudes del racismo aversivo se tienden a expresar de forma que no afecten a la autoimagen, por lo que se encauzarán como "en favor de los blancos" y no "contra los negros".
Esto se ejemplifica bien en un estudio experimental que realizaron Gaertner y Dovidio (1977) y que ha sido ampliamente citado. Un grupo de participantes blancos tenían la oportunidad de ayudar a una víctima: en un caso un blanco y en otro caso, un negro.

En Psicología Social se ha estudiado que las personas tienden menos a ayudar cuando hay muchos espectadores, dado que la responsabilidad se diluye en la multitud. En el experimento de Garertner y Dovidio se demostró que el grupo de blancos tendía más a ayudar a ambas víctimas cuando eran pocos espectadores, y a ayudar más al blanco que al negro cuando había muchos espectadores.

\section{Infra-humanización}

Recientemente, se ha desarrollado el concepto de infra-humanización para definir una nueva forma de racismo (Leyens et al, 2003). En estos casos, las personas tienden a infrahumanizar en la medida que le atribuyen una mayor esencia humana al grupo de pertenencia que a grupos externos. En estos casos, el endogrupo se atribuye a sí mismo la esencia humana, a la vez que se considera al exogrupo menos humano.

Leyens et al, (2000) hallaron que la esencia humana puede concentrarse en tres características: inteligencia, lenguaje, y emociones humanas. Por lo que, un grupo categorizado de Infra-humano lo será si se le atribuye la ausencia de alguna de estas tres categorías.

La característica de emociones humanas es la de mayor interés para esta Teoría. Para ello, se distingue entre emociones primarias y secundarias. Las emociones primarias son aquellas que comparten animales y humanos (por ejemplo, miedo), en tanto las secundarias son exclusivamente experimentadas por humanos (por ejemplo, culpa; Ekman, 1992).

La mayoría de las investigaciones en infra-humanización se ha concentrado en la atribución de emociones secundarias entre grupos, dado que es más independiente de los factores socioculturales que las otras dos características. En este sentido, diferentes estudios han demostrado que la gente tiende a atribuir mayor esencia humana al grupo de 
pertenencia que a grupos externos, basados en la mayor o menor atribución emociones secundarias (Demoulin et al, 2004; Leyens et al., 2003; 2001; Paladino et al, 2002). A modo de ejemplo, Leyens et al. (2001) solicitaron a los participantes de su estudio que seleccionaran las características que percibieran como típicas de su endogrupo y las del exogrupo. El resultado fue que la gente seleccionaba más las emociones secundarias como características de su grupo que del exogrupo.

Las situaciones que favorecen el desarrollo de la infra-humanización son:

1. aquellos individuos que se identifican fuertemente con el endogrupo (Paladino et al, 2004)

2. aquellos individuos que diferencian a los grupos en términos de esencia humana

3. aquellos individuos incluidos en una categoría que perciben como más significativa (Demoulin et al, 2009)

Este último aspecto, es el que está recibiendo mayor atención en la actualidad, y tal como demuestran Demoulin et al (2009) la infra-humanización no es solo efecto de un sesgo de favoritismo endogrupal producto de la categorización, sino que ocurre en contextos en los que las categorías sociales son percibidas como significativas. Esto es, en la génesis de la Infra-humanización no basta únicamente con la categorización sino que el significado es necesario, y la misma se completa con la atribución al endogrupo de emociones secundarias y la negación de las mismas en el exogrupo. O sea, esta forma de racismo es la tendencia de percibir al grupo propio como más humano que el otro grupo.

\section{Ontologización}

Otra de las nuevas formas de racismo identificada recientemente es la ontologización. Este concepto ha sido aplicado al racismo como una forma de inferiorizar al exogrupo, al separar a determinados grupos humanos de su propia especie y anclarlos en la naturaleza animal (Roncarati et al, 2009). La idea que subyace a esta forma de racismo es la de considerar la diferencia entre determinados grupos humanos no solo en términos culturales, sino también naturales.
Esta Teoría se basa en el concepto de ontologización desarrollado por Pérez et al., (2007) que sostienen que los grupos viven en constante contacto, sienten la necesidad de desarrollar sistemas de creencias y Prejuicios que eviten mezclas sociales.

De esta forma, Roncarati et al (2009), perciben que dentro de los diferentes contextos sociales, el de las relaciones sexuales es de especial interés para el estudio de esta nueva forma de racismo. Basados en el estudio de Kalmijn (1998) que demuestra que, en Estados Unidos, los matrimonios interraciales son menos frecuentes que entre diferentes nacionalidades o religiones, se preguntan si esto puede deberse a que existe una representación social de que las parejas interraciales "van contra la naturaleza".

Su estudio experimental demuestra un mayor grado de ontologización observado en las condiciones que se corresponden con un relacionamiento sexual interracial, y menor para el caso intra-racial (Roncarati et al, 2009).

La explicación que hallan los autores es la de explicar el relacionamiento interracial como un comportamiento impulsivo y salvaje, lo cual centra la explicación en términos biológicos y no culturales.

Este trabajo es muy importante para los estudios del nuevo racismo, porque demuestra cómo prevalece el mito de pureza racial y el miedo a la contaminación, así como el contacto entre grupos percibidos como diferentes persiste como tabú, a pesar de que en la actualidad esto no se expresa de forma directa.

\section{Hetero-etnización}

Por último, Vala et al (2009), han desarrollado el concepto de hetero-etnización, para describir una nueva forma de racismo que se caracteriza por la atribución de marcadas diferencias culturales al exogrupo.

Los autores distinguen los tres conceptos de infra-humanización, ontologización y heteroetnización. Mientras las hetero-etnización representa la exagerada atribución de diferencias culturales al exogrupo, la ontologización atribuye más rasgos naturales que culturales 
al exogrupo, y la infra-humanización niega la capacidad del exogrupo de expresar emociones secundarias.

La diferencia cultural no es un antecedente del Prejuicio, sino una expresión misma de Prejuicio. Pettigrew y Meertens (1995) mostraron a partir de una encuesta llevada a cabo en 5 países europeos que, la simple afirmación de diferencias culturales entre el endogrupo (nacionalidad europea) y el exogrupo (inmigrante no europeo), era parte de un repertorio de creencias que denominaron "Prejuicio sutil". Lo más significativo que demostraron Pettigrew y Meertens (1995) es que cuando se atribuye una diferencia -real o imaginada- a un exogrupo minoritario, esto se correlaciona con un patrón de representaciones negativas de ese exogrupo.

Lo que Vala et al (2009), notaron es que la gente Prejuiciosa es más propensa a notar diferencias -aún cuando no existen- entre el endogrupo y el exogrupo. De esta forma, la hetero-etnización atribuye una cultura inferior al exogrupo, que se encubre como una posición más socialmente aceptada de diferencia cultural. Según el estudio empírico de estos autores, la atribución de diferencias culturales a personas negras, correlacionó positivamente con evaluaciones negativas de gente negra y con la creencia de que, los grupos humanos están jerárquicamente organizados en términos biológicos y culturales (Vala et al, 2009).

\section{CONCLUSIONES}

En contra de la creencia de que el racismo está en declive en nuestras sociedades, los avances en Psicología Social demuestran que el racismo está en constante transformación, y ha encontrado la manera de adaptarse a las normativas antirracistas para subsistir.

Lo interesante es que esta adaptación se ha logrado mediante expresiones aparentemente legítimas y correctas que, como hemos visto, encubren una persistente producción de Prejuicios raciales. Principalmente, la dificultad de identificar el nuevo racismo estriba en que utiliza los valores propios de las sociedades liberales, tales como el individualismo, la libertad o la igualdad para ejercitar la discriminación.
Tal como menciona Augoustinos (2009), el definir al racismo de forma consensuada ya no refleja las múltiples formas que éste adopta en la actualidad para ejercer el Prejuicio y la discriminación, por lo que es imperativo -como psicólogos sociales- reconocer su naturaleza contradictoria, multifacética y ambivalente y por lo tanto teorizar sobre "múltiples racismos".

El movimiento que se ha realizado del concepto de raza al de cultura, puede llevar implícito el relevo de pureza racial por identidad cultural.

Mientras las nuevas formas de racismo continúen existiendo, no se habrán realizado verdaderos avances en el campo de la lucha contra el racismo y la discriminación racial y étnica. Por lo tanto, más que reprimir la expresión del racismo, es necesaria la educación en contextos multiculturales, aunque primeramente sea preciso hacer una concientización del propio racismo.

\section{REFERENCIAS}

Aberson, C. L., Healy, M., y Romero, V. (2000). Ingroup bias and self-esteem: A meta-analysis. Personality and Social Psychology Review, 4, 157-173.

Aboud, F. E. (1980). A test of ethnocentrism with young children. Canadian Journal of Behavioral Science, 12, 195-209.

Allport, F., Allport, G., Brown, J., Cantril, H., Doob, L., y English, H., (1939). Resolution and manifestoes of scientists. Science, 89, 166-169.

Allport, G.W. (1954). The nature of Prejudice. Reading, Mass. Addison-Wesley.

Anton, J. y Del Popolo, F. (2008). Visibilidad estadística de la población afrodescendiente de América Latina: aspectos conceptuales y metodológicos. Santiago de Chile: CEPAL/SEGIB.

Augoustinos, M. (2009). Racism(s): one or many? International Journal of Psychology, 44, 1-3.

Augoustinos, M. y Reynolds, K. J. (2001). Prejudice, Racism, and Social Psychology. En Augoustinos y K. J. Reynolds (Eds.), Understanding prejudice, racism, and social psychology (pp 1-23). London: Sage.

Banco Mundial (2004) Desigualdad en América Latina y el Caribe ¿Ruptura con la historia?. Disponible en www. bancomundial.org.

Banco Mundial (2008). Report: Outsiders? The changing patterns of exclusion in Latin America and the Caribbean. Washington, DC.: WB.

Bello, A. y Paixao, A. (2008). Estado actual del cumplimiento de los derechos civiles, políticos, económicos, sociales y culturales de la población afro-descendiente en América Latina. Santiago de Chile: CEPAL. 
Billig, M. (1998, July). A dead idea that won't lie down. Searchlight. Retrieved March 20, 2008 from www. searchlightmagazine.com.

Brewer, M. B. (1979). In-group bias in the minimal intergroup situation: A cognitive-motivational analysis. Psychological Bulletin, 86, 307-324.

Brewer, M. B. y Campbell, D. T. (1976). Ethnocentrism and intergroup attitudes: East African evidence. New York: Sage.

Brewer, M.B. (1999). The psychology of prejudice: Ingroup love or outgroup hate? Journal of Social Issues, 55, 429-444.

Brown, R. (1995). Prejudice: its social psychology. Oxford, UK: Blackwell.

Campbell, D. T. (1965). Ethnocentric and other altruistic motives. En D. Levine (Ed.), Nebraska Symposium on Motivation, Vol. 13, (pp. 283-311). Lincoln: University of Nebraska Press.

Demoulin, S., Cortes, B., Viki, G., Rodríguez, A., Rodríguez, R., Paladino, M., y Leyens, J. Ph. (2009). The role of in-group identification in nfrahumanization. International Journal of Psychology, 44, 4-11.

Demoulin, S., Rodriguez, R. T., Rodriguez, A. P., Vaes, J., Paladino, M. P., Gaunt, R., Cortes, B. P., y Leyens, J. Ph. (2004). Emotional prejudice can lead to infrahumanization. En W. Stroebe y M. Hewstone (Eds.), European review of social psychology, Vol. 15 (pp. 259-296). Hove, UK: Psychology Press.

Dion, K. (2002). The social psychology of perceived prejudice and discrimination. Canadian Psychology, 43, 1-10.

Dovidio, J. F. (2001). On Contemporary Prejudice: The Third Wave. Journal of Social Issues, 57, $829-849$

Dovidio, J. F., Smith, J. K., Donella, A. G., y Gaertner, S. L. (1997). Racial attitudes and the death penalty. Journal of Applied Social Psychology, 27, 1468-1487.

Dovidio, J. F., y Gaertner, S. (2000). Aversive racism and selection decisions: 1989 and 1999. Psychological Science, 11, 315-319.

Dovidio, J., y Fazio, R. (1992). New technologies for the direct and the indirect assessment of attitudes. En J. Tanur (Ed.), Questions about questions: Inquiries into the cognitive bases of surveys (pp. 204-237). New York: Sage.

Duckitt, J. (2001). A dual-process cognitive-motivational theory of ideology and prejudice. En M. P. Zanna, (Ed.), Advances in experimental social psychology, (Vol. 33, pp. 41-113). New York: Academic Press.

Eiser, J. R. y Stroebe, W. (1972). Categorisation and social judgment. London: Academic Press.

Ekman, P. (1992). An argument for basic emotions. Cognition and Emotion, 6, 169-200.

Fein, S. y Spencer, S. J. (1997). Prejudice as self-image maintenance: Affirming the self through derogating others. Journal of Personality and Social Psychology, 73, 31-44

Foroohar, R. (2002, Febrero). Race in the boardroom. Newsweek, 18, 34-38

Gaertner, S. L. y Dovidio, J. F. (1986). The aversive form of racism. En J. F. Dovidio y S. L. Gaertner (Eds.), Preju- dice, discrimination, and racism (pp. 61-89). Orlando, FL: Academic Press.

Gaertner, S. L. y. Dovidio, J. F. (1977). The subtlety of white racism, arousal and helping behavior. Journal of Personality and Social Psychology, 35, 691-707

Gannett, L. (2001). Racism and human genome diversity research: The ethical limits of "Population Thinking. Philosophy of Science, 68, 479-492.

Gilbert, D. T., y Hixon, J. (1991). The trouble of thinking: Activation and application of stereotypic beliefs. Journal of Personality and Social Psychology, 60, 509-517.

Giles, H (Ed. ) (1977). Language, Ethnicity and Intergroup Relations. London: Academic Press.

Gurr, T. R. (1970). Why men rebel. Princeton, NJ: PUP.

Hamilton, D. L. y Guifford, R. K. (1976). Illusory correlation in interpersonal perception: a cognitive basis of stereotypic judgements. Journal of Experimental Social Psychology, 12, 392-407.

Henry, P. J. y Sears, D. O. (2002). The Symbolic Racism 2000 Scale. Political Psychology, 23, 253-283.

Hodson, G., Dovidio, J. F., y Gaertner, S. L. (2002). Processes in racial discrimination: Differential weighting of conflicting information. Personality and Social Psychology Bulletin, 28, 460-471.

Jackson, J. S., Brown, K. T., y Kirby, D. C. (1998). International perspectives on prejudice and racism. En J. L. Eberhardt y S. T. Fiske (Eds.), Confronting racism: The problem and the response (pp. 101-135). Thousand Oaks, CA: Sage.

Kalmijn, M. (1998). Intermarriage and homogamy: Causes, patterns, trends. Annual Review of Sociology, 24, 395-421.

Katz, I. (1981). Stigma: A social psychological analysis. Hillsdale, NJ: Erlbaum.

Kinder, D., y Sears, D. O. (1981). Prejudice and politics: Symbolic racism versus racial threats to the good life. Journal of Personality and Social Psychology, 40, 414-431.

Krueger, J. y Clement, R. w. (1994). Memory-based judgments about multiple categories: a revision and extension of Tajfel's Accentuation Theory. Journal of Personality and Social Psychology, 67, 35-47.

LeVine, R. A., y Campbell, D. T. (1972). Ethnocentrism: Theories of conflict, ethnic attitudes, and group behavior. New York: Wiley.

Leyens, J. Ph., Cortes, B. P., Demoulin, S., Dovidio, J., Fiske, S. T., Gaunt, R., Paladino, M. P., Rodriguez, A. P., Rodriguez, R. T., y Vaes, V. (2003). Emotional prejudice, essentialism, and nationalism. European Journal of Social Psychology, 33, 704-707.

Leyens, J. Ph., Paladino, P. M., Rodriguez, R. T., Vaes, J., Demoulin, S., Rodriguez, A. P., y Gaunt, R. (2000). The emotional side of prejudice: The role of secondary emotions. Personality and Social Psychology Review, 4, 186-197.

Linville, P. W. (1998). The heterogeneity of homogeneity. En J. M. Darley y J. Cooper (Eds), Attribution and social interaction: The legacy of Edward E. Jones (pp. 423-462). Washington, DC: American Psychological Association. 
Mackie, D. M., Queller, S., Stroessner, S. J., y Halmilton, D. L. (1996). Stereotypes better or worse: Multiple roles of positive affect in group impressions. En R. M. Sorrentino y E. T. Higgins (Eds.), Handlbook of motivation and cogntion: the interpersonal context (pp. 371-396). New York: Guilford Press.

McConahay, J. B. (1983). Modern racism and modern discrimination: the effects of race, racial attitudes, and context on simulated hiring decisions. Personality and Social Psychology Bulletin, 9, 551-558.

McConahay, J. B. (1986). Modern racism, ambivalence, and the modern racism scale. En J. F. Dovidio y S. L. Gaertner (Eds.), Prejudice, discrimination, and racism (pp. 61-89). Orlando, FL: Academic Press.

Munné, F. (1986). La construcción de la psicología social como ciencia teórica. Barcelona, Alamex.

Paladino, M. P., Vaes, J., Castano, E., Demoulin, S., y Leyens, J. Ph. (2004). Emotional infra-humanisation in intergroup relations: The role of national identities in the attribution of primary and secondary emotions to Italians and Germans. Cahiers de Psychologie Cognitive/ Current Cognitive Psychology, 22, 519-536.

Paladino, P. M., Leyens, J. Ph., Rodriguez, R. T., Rodriguez, A. P., Gaunt, R., y Demoulin, S. (2002). Differential association of uniquely and nonuniquely human emotions to the in-group and the out-groups. Group Processes and Intergroup Relations, 5, 105-117.

Pascale, P. (2008). La creatividad: un análisis de la literatura científica publicada y del impacto de los modelos de confluencia. Trabajo presentado en el Congreso Internacional de Innovación y Creatividad, Octubre, Loulé.

Pérez, J. A., Moscovici, S., y Chulvi, B. (2007). The taboo against group contact. Hypothesis of Gypsy ontologization. British Journal of Social Psychology, 46, 249-272.

Pettigrew, T. F. (1998). Prejudice and discrimination on the college campus. En J. L. Eberhardt y S. T. Fiske (Eds.), Confronting racism: The problem and the response (pp. 263-279). Thousand Oaks, CA: Sage.

Pettigrew, T. F., Christ, O., Wagner, U., Meertens, R. W., van Dick, R. y Zick, A. (2008). Relative Deprivation and Intergroup Prejudice. Journal of Social Issues. 64, 385-401.

Pettigrew, T. F., y Meertens, R. W. (1995). Subtle and blatant prejudice in Western Europe. European Journal of Social Psychology, 25, 57-75.
Richards, G. (1997). Race, racism and psychology: Towards a reflexive history. New York: Routledge.

Roncarati, A., Pérez, J. A., Ravenna, M., \& Navarro-Pertusa, E. (2009). Mixing against culture vs mixing against nature: Ontologization of prohibited interethnic relationships. International Journal of Psychology, 44, 12-19.

Sears, D. O. (1988). Symbolic racism. En P.A. Katz y D.A. Taylor (Eds.), Eliminating racism: Profiles in controversy (pp. 53-84). New York: Plenum.

Stephan, W. G., y Stephan, C. W. (1985). Intergroup anxiety. Journal of Social Issues, 41, 157-175.

Tajfel, H. (1959). Quantitative judgment in social perception. British Journal of Psychology, 50, 16-29

Tajfel, H. y Fraser, C. (1978). Introducing social psychology. Harmondsworth: Penguin Books.

Tajfel, H. y Turner, J. C. (1986). The social identity theory of inter-group behavior. In S. Worchel y L. W. Austin (Eds.), Psychology of Intergroup Relations. Chigago: Nelson-Hall

Tajfel, H. y Wilkes, A. L. (1963). Clasiffication and quantitative judgment. British Journal of Psychology, 54, 101-114.

Thompson, S. K. (1975). Gender labels and early sex-role development. Child Development, 46, 339-347.

Vala, J. (2009). Editorial: Expressions of "new" racism. International Jorunal of Psychology. 44, 1-3.

Vala, J., Pereira, C., \& Costa-Lopes, R. (2009). Is the attribution of cultural differences to minorities an expression of racial prejudice? International Journal of Psychology, $44,20-28$.

Walker, I. (2001). The changing nature of racism: From old to new?. En Augoustinos y K. J. Reynolds (Eds.), Understanding prejudice, racism, and social psychology (pp 24-42). London: Sage.

Wilder, D. A., \& Shapiro, P. N. (1999). Effects of anxiety on impression formation in a group context: An anxietyassimilation hypothesis. Journal of Experimental Social Psychology, 25, 481-499.

Williams, J. E. y Morland, J. K. (1976). Race, colour and the young child. Chapel Hill: UNC Press.

Yee, M. D. y Brown, R. (1994). The development of gender differentiation in young vhildern. British Journal of Social Psychology, 33, 183-196.

Zack, N. (2002) Race and Racial Discrimination. En H. Lafollette (ed), Oxford Hand Book of Practical Ethics. Oxford University Press, pp. 245-271. 


\section{APÉNDICE 1. \\ TENDENCIA EXPONENCIAL DE PUBLICACIONES EN PREJUICIO Y DISCRIMINACIÓN}

Se especifica un modelo exponencial para la tendencia temporal donde se incorpora un término de perturbación viene dada por:

$$
y_{t}=\beta_{0} e^{\beta_{1} t+\varepsilon_{t}}
$$

\section{Donde}

$y_{t}$ es la serie anual de publicaciones; $\beta_{0}$ término de escala, constante o término independiente; $\beta_{1}$ coeficiente del término exponencial.

De manera que si aplicamos logaritmo neperiano a la anterior expresión a ambos lados de la igualdad tenemos que:

$$
\ln y_{t}=\ln \beta_{0}+\beta_{1} t+\varepsilon_{t} 1
$$

Resultados de la estimación:

Dependent Variable: LOG(PUBLICACIONES) Method: Least Squares

Sample: 19802007

Included observations: 28

\begin{tabular}{lllll}
\multicolumn{1}{c}{ Variable } & Coefficient & Std. Error & t-Statistic & Prob. \\
\hline \hline \multicolumn{1}{c}{ C } & 3.797370 & 0.083842 & 45.29179 & 0.0000 \\
\multicolumn{1}{c}{ @TREND } & 0.072776 & 0.005329 & 13.65565 & 0.0000 \\
\hline \hline R-squared & 0.877634 & Mean dependent var & 4.779846 \\
Adjusted R-squared & 0.872927 & S.D. dependent var & 0.639027 \\
S.E. of regression & 0.227796 & Akaike info criterion & -0.051988 \\
Sum squared resid & 1.349161 & Schwarz criterion & 0.043170 \\
Log likelihood & 2.727826 & F-statistic & 186.4767 \\
Durbin-Watson stat & 0.644975 & Prob(F-statistic) & 0.000000 \\
\hline \hline
\end{tabular}

\section{Donde:}

$$
\ln y_{t}=\underset{(0.083842)}{3.79737}+\underset{(0.005329)}{0.072776 t}+\varepsilon_{t} 2
$$

Siendo ambos significativos, al igual que el modelo en su conjunto, con un confianza del $99 \%$. El R cuadrado ajustado del modelo nos indica que el modelo es capaz de explicar un $87.29 \%$ de la variabilidad temporal de la serie.

Podemos ver el ajuste del modelo a partir del siguiente gráfico, donde se presenta la serie de publicaciones y la predicha exclusivamente a partir de una trayectoria intertemporal exponencial.

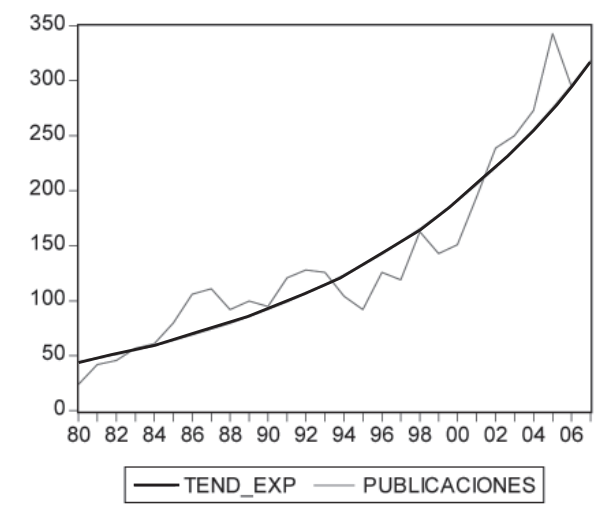

